\title{
Multi-band transit observations of the TrES-2b exoplanet ${ }^{\star}$
}

\author{
D. Mislis ${ }^{1}$, S. Schröter ${ }^{1}$, J. H. M. M. Schmitt ${ }^{1}$, O. Cordes $^{2}$, and K. Reif ${ }^{2}$ \\ 1 Hamburger Sternwarte, Gojenbergsweg 112, 21029 Hamburg, Germany \\ e-mail: mdimitri@hs.uni-hamburg.de \\ 2 Argelander-Institut für Astronomie, Auf dem Hügel 71, 53121 Bonn, Germany \\ Received 17 July 2009 / Accepted 14 December 2009
}

\begin{abstract}
We present a new data set of transit observations of the TrES-2b exoplanet taken in spring 2009, using the $1.2 \mathrm{~m}$ Oskar-Lühning telescope (OLT) of Hamburg Observatory and the $2.2 \mathrm{~m}$ telescope at Calar Alto Observatory using BUSCA (Bonn University Simultaneous CAmera). Both the new OLT data, taken with the same instrumental setup as our data taken in 2008, as well as the simultaneously recorded multicolor BUSCA data confirm the low inclination values reported previously, and in fact suggest that the TrES-2b exoplanet has already passed the first inclination threshold $\left(i_{\min , 1}=83.417^{\circ}\right)$ and is not eclipsing the full stellar surface any longer. Using the multi-band BUSCA data we demonstrate that the multicolor light curves can be consistently fitted with a given set of limb darkening coefficients without the need to adjust these coefficients, and further, we can demonstrate that wavelength dependent stellar radius changes must be small as expected from theory. Our new observations provide further evidence for a change of the orbit inclination of the transiting extrasolar planet TrES-2b reported previously. We examine in detail possible causes for this inclination change and argue that the observed change should be interpreted as nodal regression. While the assumption of an oblate host star requires an unreasonably large second harmonic coefficient, the existence of a third body in the form of an additional planet would provide a very natural explanation for the observed secular orbit change. Given the lack of clearly observed short-term variations of transit timing and our observed secular nodal regression rate, we predict a period between approximately 50 and 100 days for a putative perturbing planet of Jovian mass. Such an object should be detectable with present-day radial velocity (RV) techniques, but would escape detection through transit timing variations.
\end{abstract}

Key words. planetary systems - techniques: photometric - stars: individual: TrES-2b

\section{Introduction}

As of now more than 400 extrasolar planets have been detected around solar-like stars. In quite a few cases several planets have been detected to orbit a given star, demonstrating the existence of extrasolar planet systems in analogy to our solar system. Just as the planets in our solar system interact gravitationally, the same must apply to extrasolar planet systems. Gravitational interactions are important for the understanding of the long-term dynamical stability of planetary systems. The solar system has been around for more than four billion years, and the understanding of its dynamical stability over that period of time is still a challenge Simon et al. (1994). In analogy, extrasolar planet systems must be dynamically stable over similarly long time scales, and most stability studies of extrasolar planet systems have been directed towards an understanding of exactly those long time scales. Less attention has been paid to secular and short-term perturbations of the orbit, since such effects are quite difficult to detect observationally. Miralda-Escudé (2002) gives a detailed discussion on what secular effects might be derivable from extrasolar planet transits; in spectroscopic binaries the orbit inclination $i$ can only be deduced in conjunction with the companion mass, and therefore the detection of an orbit inclination change is virtually impossible. Short-term transit timing variations (TTVs) have been studied by a number of authors (Holman \& Murray 2005; Agol et al. 2005), however, a detection of such effects has remained

* Photometric transit data are only available in electronic form at the CDS via anonymous ftp to

cdsarc.u-strasbg.fr $(130.79 .128 .5)$ or via

http://cdsweb.u-strasbg.fr/cgi-bin/qcat?]/A+A/510/A107 elusive so far. In principle, a detection of orbit change would be extremely interesting since it would open up entirely new diagnostic possibilities of the masses and orbit geometries of these systems; also, in analogy to the discovery of Neptune, new planets could in fact be indirectly detected.

The lightcurve of a transiting extrasolar planet with known period allows very accurate determinations of the radii of star and planet (relative to the semi-major axis) and the inclination of the orbital plane of the planet with respect to the line of sight towards the observer. Clearly, one does not expect the sizes of host star and planet to vary on short time scales, however, the presence of a third body can change the orientation of the orbit plane and, hence, lead to a change in the observed inclination with respect to the celestial plane. The TrES-2 exoplanet is particularly interesting in this context. It orbits its host star once in 2.47 days, which itself is very much solar-like with parameters consistent with solar parameters; its age is considerable and, correspondingly, the star rotates quite slowly. Its close-in planet with a size of $1.2 R_{\text {Jup }}$ is among the most massive known transiting extrasolar planets (Holman et al. 2007; Sozzetti et al. 2007).

What makes the TrES-2 exoplanet orbits even more interesting, is the fact that an apparent inclination change has been reported by some of us in a previous paper (Mislis \& Schmitt 2009, henceforth called Paper I). The authors carefully measured several transits observed in 2006 and 2008. Assuming a circular orbit with constant period $P$, the duration of an extrasolar planet transit in front of its host star depends only on the stellar and planetary radii, $R_{\mathrm{s}}$ and $R_{\mathrm{p}}$, and on the inclination $i$ of the orbit plane w.r.t. the sky plane. A linear best fit to the currently available inclination measurements yields an apparent 
Table 1. Observation summary.

\begin{tabular}{lllll}
\hline \hline Date & Instrument & Filter & Airmass & Seeing \\
\hline $11 / 04 / 2009$ & OLT & $I$ & $1.7877-1.0712$ & $2.94^{\prime \prime}$ \\
$28 / 05 / 2009$ & BUSCA & $v, b, y, I$ & $1.8827-1.0453$ & $3.09^{\prime \prime}$ \\
\hline
\end{tabular}

inclination decrease of $5.1 \times 10^{-4} \mathrm{deg} / \mathrm{day}$. The transit modeling both by Holman et al. (2007) and Mislis \& Schmitt (2009) shows the transit of TrES-2b in front of its host star to be "grazing". In fact, according to the latest modeling the planet occults only a portion of the host star and transits are expected to disappear in the time frame 2020-2025, if the observed linear trend continues. This "grazing" viewing geometry is particularly suitable for the detection of orbital changes, since relatively small changes in apparent inclination translate into relatively large changes in eclipse duration. At the same time, a search for possible TTVs by Rabus et al. (2009) has been negative; while Rabus et al. (2009) derive a period wobble of $57 \mathrm{~s}$ for TrES-2, the statistical quality of their data is such that no unique periods for TTVs can be identified.

The purpose of this paper is to present new transit observations of the TrES-2 exoplanet system obtained in 2009, which are described in the first part (Sect. 2) and analysed (Sect. 3). In the second part (Sect. 4) of our paper we present a quantitatively analysis of what kind of gravitational effects can be responsible for the observed orbit changes of TrES-2b and are consistent with all observational data of the TrES-2 system.

\section{Observations and data reduction}

We observed two transits of TrES-2 using the ephemeris suggested by O'Donovan et al. (2006) and by Holman et al. (2007) from $T_{\mathrm{c}}(E)=2453957.6358[\mathrm{HJD}]+E \cdot(2.47063$ days $)$, using the $1.2 \mathrm{~m}$ Oskar Lühning telescope (OLT) at Hamburg Observatory and Calar Alto Observatory $2.2 \mathrm{~m}$ telescope with BUSCA.

The OLT data were taken on 11 April 2009 using a $3 K \times 3 K$ CCD with a $10^{\prime} \times 10^{\prime}$ FOV and an I-band filter as in our previous observing run (Paper I). The readout noise and the gain were $16.37 \mathrm{e}^{-}$and $1.33 \mathrm{e}^{-} /$ADU respectively. With the OLT we used 60-s exposures which provided an effective time resolution of $1.17 \mathrm{~min}$. During the observation, the airmass value ranged from 1.7877 to 1.0712 and the seeing was typically $2.94^{\prime \prime}$.

The Calar Alto data were taken on 28 May 2009 using BUSCA and the $2.2 \mathrm{~m}$ telescope. BUSCA is designed to perform simultaneous observations in four individual bands with a FOV of $11^{\prime} \times 11^{\prime}$. Therefore it has four individual $4 \mathrm{~K} \times 4 \mathrm{~K}$ CCD systems which cover the ultra-violet, the blue-green, the yellow-red and the near-infrared part of the spectrum (channel a-d respectively). For this run we used the Strömgren filters v (chn. a), b (chn. b), and y (chn. c), and a Cousins- $I$ filter for the near-infrared (chn. d). The readout-noise for these four detectors are $9.09 \mathrm{e}^{-}, 3.50 \mathrm{e}^{-}, 3.72 \mathrm{e}^{-}$, and $3.86 \mathrm{e}^{-}$respectively for the $\mathrm{a}, \mathrm{b}, \mathrm{c}$, and $\mathrm{d}$ channels. The gain values for the same channels are $1.347 \mathrm{e}^{-} /$ADU, $1.761 \mathrm{e}^{-} /$ADU, $1.733 \mathrm{e}^{-} / \mathrm{ADU}$, and $1.731 \mathrm{e}^{-} / \mathrm{ADU}$ respectively. The airmass value ranged from 1.8827 to 1.0453 and the seeing was $3.09^{\prime \prime}$. For the BUSCA observations we took $30 \mathrm{~s}$ exposure yielding an effective time resolution of $1.63 \mathrm{~min}$. In Table 1 we summarize the relevant observation details.

For the data reduction, we used Starlink and DaoPhot software routines, and the MATCH code. We perform the normal
Table 2. Relative photometry data.

\begin{tabular}{llll}
\hline \hline HJD & RelativeFlux & Uncertainty & Flag \\
\hline 2454933.44031 & 0.99172 & 0.0037 & $O L T-I$ \\
2454933.44091 & 0.99204 & 0.0038 & $O L T-I$ \\
2454933.44221 & 1.00514 & 0.0039 & $O L T-I$ \\
2454933.44281 & 1.00314 & 0.0038 & $O L T-I$ \\
2454933.44341 & 0.99458 & 0.0035 & $O L T-I$ \\
2454933.44401 & 0.99745 & 0.0037 & $O L T-I$ \\
\hline
\end{tabular}

Notes. Relative photometry vs. time; note that the complete table is available only electronically at the CDS. The time stamps refer to the Heliocentric Julian Date (HJD) at the middle of each exposure. The "Flag" column refers to the telescope and filter.

reduction tasks, bias subtraction, dark correction, and flat fielding on the individual data sets before applying aperture photometry on on all images. For TrES-2, we selected the aperture photometry mode using apertures centered on the target star, check stars, and sky background. Typical sky brightness values for the 11 April and for the 28 May were 89 and 98 ADUs, respectively, i.e., values at a level $0.008 \%$ and $0.006 \%$ of the star's flux, respectively (for $I$-filter). For the relative photometry we used the star U1350-10220179 as a reference star to test and calibrate the light curve. For the data analysis presented in this paper we did not use additional check stars, but note that we already checked this star for constancy in the Paper I. To estimate the magnitude errors, we followed Howell \& Everett (2001) and the same procedure as described in our first paper (Paper I) to obtain better relative results. Our final relative photometry is presented in Table 2, which is available in its entirety in machine-readable form in the electronic version of this paper.

\section{Model analysis and results}

In our model analysis we proceeded in exactly the same fashion as described in Paper I. Note that the assumption of circularity appears to apply very well to TrES-2b (O'Donovan et al. 2009; O'Donovan et al. 2006); the assumption of constant period and hence constant semi-major axis will be adressed in Sect. 4. For our modelling we specifically assumed the values $R_{\mathrm{S}}=1.003 R_{\mathrm{O}}$, $R_{\mathrm{p}}=1.222 R_{\mathrm{J}}, P=2.470621$ days, $\alpha=0.0367 \mathrm{AU}$ for the radii of star and planet, their period and the orbit radius respectively. All limb darkening coefficients were taken from Claret (2004), and for the OLT data we used the same values as in Paper I, viz., $u_{1}=0.318$ and $u_{2}=0.295$ for the $I$ filter, as denoted by $\mathrm{S} 1$ in Table 3.

\subsection{OLT data and modeling}

Our new OLT data from 11 April 2009 were taken with the same instrumental setup as our previous data taken on 18 September 2008. The final transit light curve with the best fit model is shown in Fig. 1, the reduced light curve data are provided in Table 1. Keeping the planetary and stellar radii and the limb darkening coefficients fixed (at the above values), we determine the best fit inclination and the central transit time $T_{\mathrm{c}}$ with our transit model using the $\chi^{2}$ method; the thus obtained fit results are listed in Table 3. The errors in the derived fit parameters are assessed with a bootstrap method explained in detail in Paper I, however, we do not use random residuals for the new model, but we circularly shift the residuals after the model substraction to produce new light curves for the bootstrapped data following 
Table 3. Individual values of duration, inclination, $\chi^{2}$ values and limb darkening coefficients from five light curve fits.

\begin{tabular}{|c|c|c|c|c|c|c|}
\hline$T_{\mathrm{c}}$ time [HJD] & & & & $\operatorname{Er}$ & $\chi^{2} \mathrm{ve}$ & I \\
\hline $3989.7529 \pm 0.00069$ & 110.308 & 0.432 & 83.59 & 0.019 & 432.1 & \\
\hline 066 & & 448 & 3.56 & .019 & 29 & \\
\hline 00070 & 109.025 & 0.430 & 3.55 & 0.019 & 290.6 & \\
\hline 0072 & 106.620 & 0.883 & 83.44 & 0.036 & 179.1 & \\
\hline $4728-r-r-2$ & 106 & 0.870 & 83.43 & 0.036 & 190.1 & \\
\hline \pm 0.00076 & 105.077 & 0.964 & 83.38 & 0.039 & 296.1 & $S 1$ \\
\hline 068 & 105 & 0.8 & 83.38 & 0. & 18 & $S$ \\
\hline 090 & 104 & 1.076 & 83.37 & 0.043 & 202.2 & $S 2$ \\
\hline 082 & 103 & 1.021 & 83.33 & 0.041 & 186.0 & $S$ \\
\hline 498 & 104 & 1.194 & 83.35 & 0.048 & 195.0 & $S 4$ \\
\hline $4980.4675 \pm 0.00060$ & 104.522 & & 83.36 & 0.030 & & \\
\hline
\end{tabular}

Notes. Units for duration and errors are minutes and for inclination errors are degrees. The OLT light curve has 268 points, the $I$-filter light curve has 198, the $y$-filter light curve has 208 , the $b$-filter light curve 198 and the $v$-filter light curve 162 points respectively.

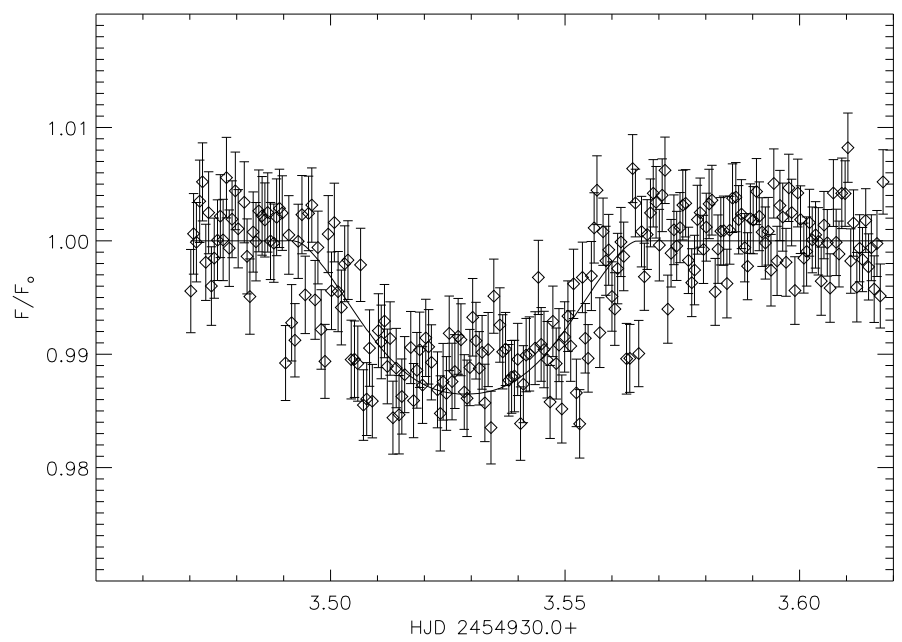

Fig. 1. Observed TrES-2 light curves and model fits for the light curve taken with the 1.2 m OLT at Hamburg Observatory taken in April 2009.

Alonso et al. (2008). In Table 3 we also provide corrected central times and errors for those transists already reported in Paper I, since due to some typos and mistakes the numbers quoted for central time and their error are unreliable (five first lines of Table 3). In Fig. 3 we plot the thus obtained inclination angle distribution for epoch April 2009 (solid curve) compared to that obtained in September 2008 (dashed curve). While the best fit inclination of $i=83.38^{\circ}$ differs from that measured in September $2008\left(i=83.42^{\circ}\right)$, the errors are clearly so large that we cannot claim a reduction in inclination from our OLT data (taken with the same instrumental setup, i.e., in September 2008 and April 2009) alone, however, is clearly consistent with such a reduction.

\subsection{BUSCA data and modeling}

Our BUSCA data have the great advantage of providing simultaneously measured multicolor data, which allows us to demonstrate that limb darkening is correctly modelled and does not affect the fitted inclinations and stellar radii. The limb darkening coefficients used for the analysis of the BUSCA data were also taken from Claret (2004); we specifically used $u_{1}=0.318$ and $u_{2}=0.295$ for the $I$ filter $(\mathrm{S} 1)$, i.e., the same values as for the OLT, $u_{1}=0.4501$ and $u_{2}=0.286$ for the $y$ filter (S2),

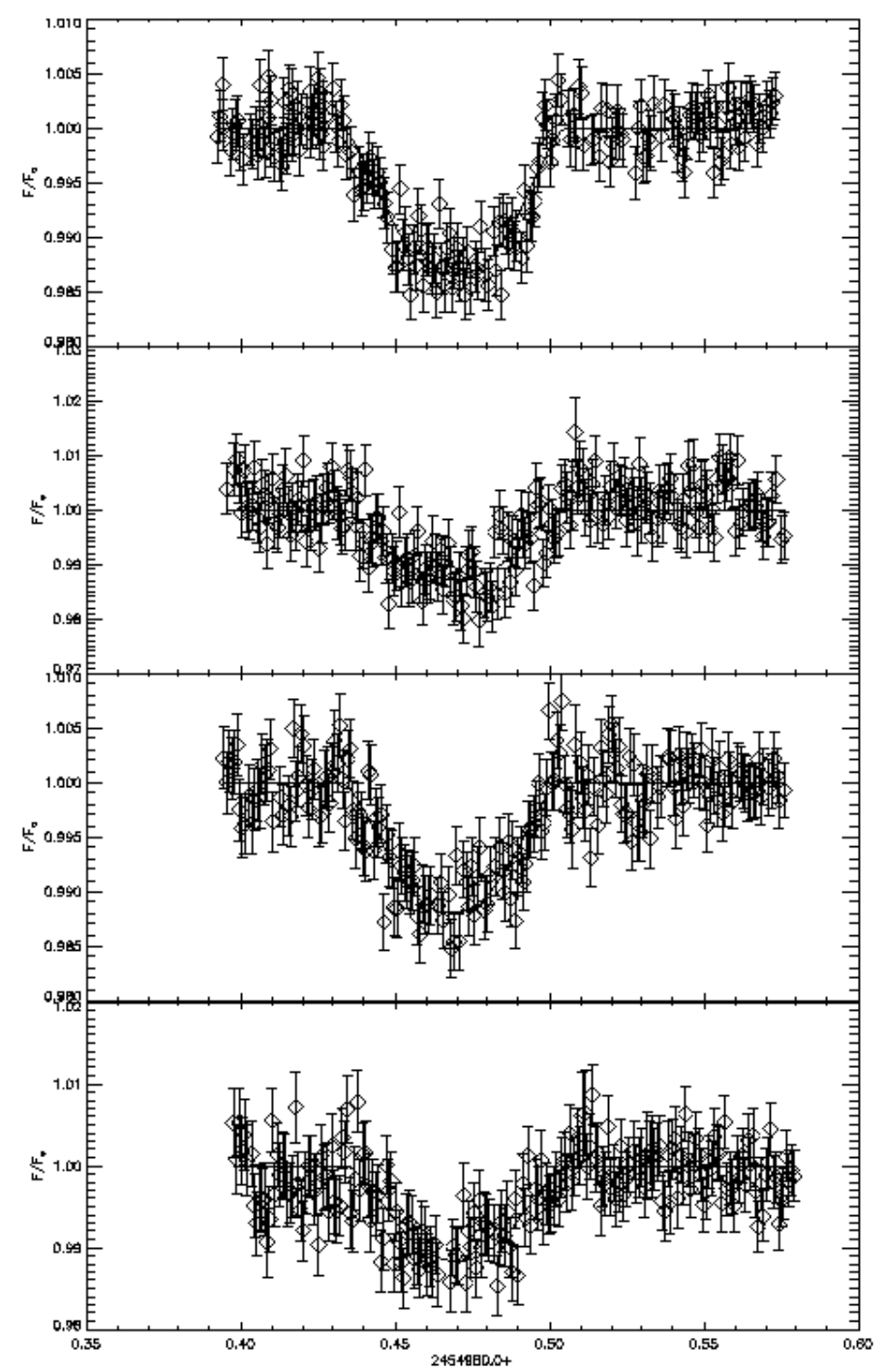

Fig. 2. Four light curves and corresponding model fits taken in May 2009 with the $2.2 \mathrm{~m}$ telescope at Calar Alto using BUSCA.

$u_{1}=0.5758$ and $u_{2}=0.235$ for the $b$ filter (S3), $u_{1}=0.7371$ and $u_{2}=0.1117$ for the $v$ filter (S4) respectively. The data reduction and analysis was performed in exactly the same fashion as for the OLT data, we also used the same comparison star U1350-10220179; the reduced light curve data are also provided in Table 2. The modelling of multicolor data for extrasolar planet transits needs some explanation. In our modelling the host star's radius is assumed to be wavelength independent. Since stars do not have solid surfaces, the question arises how much the stellar radius $R_{*}$ does actually change with the wavelength. This issue has been extensively studied in the solar context, where the limb of the Sun can be directly observed as a function of wavelength (Neckel 1995). Basically the photospheric height at an optical depth of unity is determined by the ratio between pressure and the absorption coefficient, and for the Sun, Neckel (1995) derives a maximal radius change of 0.12 arcsec between 3000 and $10000 \AA$, which corresponds to about $100 \mathrm{~km}$. If we assume similar photospheric parameters in the TrES-2 host star, which appears reasonable since TrES-2 is a G0V star, we deduce a wavelength-dependent radius change on the order of $100 \mathrm{~km}$, which is far below a percent of the planetary radius and thus not detectable. Therefore in our multi-band data modelling we can 


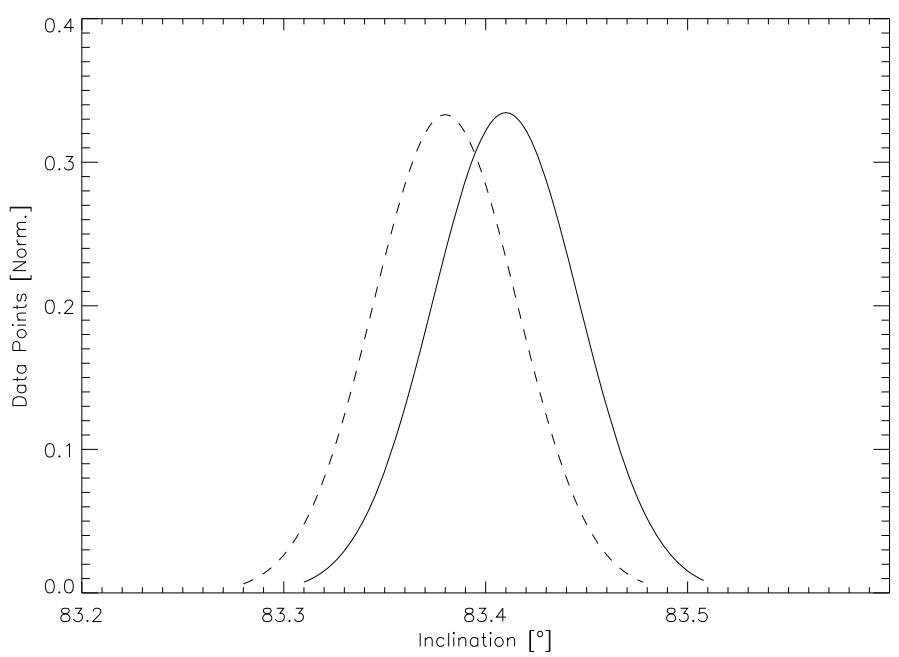

Fig. 3. Inclination distribution for the OLT data using 1000 Monte Carlo simulations for the September 2008 (solid curve) and April 2009 (dashed curve); see the text for details.

safely fix the radius of the star and the radius of the planet in a wavelength-independent fashion.

We thus kept the stellar and planetary fixed at the above values, treated all BUSCA channels as independent observations and fitted the light curves - as usual - by adjusting inclination and central transit time. The filter light curves are shown together with the so obtained model fits in Fig. 2, the model fit parameters are again summarized in Table 3. We emphasize that we obtain good and consistent fits for all light curves with the chosen set of limb darkening coefficients, thus demonstrating our capability to correctly model multicolor light curves.

Since the BUSCA data are recorded simultaneously, it is clear that the light curves in the four BUSCA channels must actually be described by the same values of inclination and transit duration. We therefore simultaneously fit all the four light curves, leaving free as fit parameters only the central time $T_{\mathrm{c}}$ and inclination $i$. With this approach we find an average inclination of $i=83.36^{\circ} \pm 0.03^{\circ}$, which is consistent with our spring OLT data and also suggests that the inclination in spring 2009 has further decreased as compared to our 2008 data.

\subsection{Joint modeling}

Using all our data we can check whether our assumption of the wavelength-independence of the radius is consistent with the observations. For this consistency test we kept the inclination value fixed at $i=83.36^{\circ}$ and fitted only the radius of the star $R_{\mathrm{s}}$ and $T_{\mathrm{c}}$. The errors on $R_{\mathrm{S}}$ were again assessed by a Monte Carlo simulation as described in Paper I and the distribution of the thus derived stellar radius values $R_{\mathrm{s}}$ is shown in Fig. 4; as apparent from Fig. 4, all BUSCA channels are consistent with the same stellar radius as - of course - expected from theory since any pulsations of a main-sequence star are not expected to lead to any observable radius changes.

The crucial issue about the TrES-2 exoplanet is of course the constancy or variability of its orbit inclination. Our new BUSCA and OLT data clearly support a further decrease in orbit inclination and hence decrease in transit duration. In order to demonstrate the magnitude of the effect, we performed one more sequence of fits, this time keeping all physical parameters fixed and fitting only the central transit times $T_{\mathrm{c}}$ using 1000 Monte Carlo realisations and studying the resulting distribution in $\chi^{2}$;
Table 4. $\chi^{2}$ tests for two different inclination values and the $\chi^{2}$ errors after 1000 Monte Carlo simulations.

\begin{tabular}{llllll}
\hline \hline Inclination [ ${ }^{\circ}$ ] & OLT & $B-I$ & $B-y$ & $B-b$ & $B-v$ \\
\hline 83.57 (Holman) & 304.9 & 199.4 & 207.8 & 216.0 & 206.3 \\
$\sigma_{\chi^{2}}$ (Holman) & 16.12 & 14.04 & 16.16 & 21.05 & 14.26 \\
83.36 (this paper) & 213.8 & 173.44 & 164.3 & 180.0 & 131.1 \\
$\sigma_{\chi^{2}}$ (this paper) & 11.96 & 11.11 & 9.96 & 12.01 & 8.77 \\
\hline
\end{tabular}

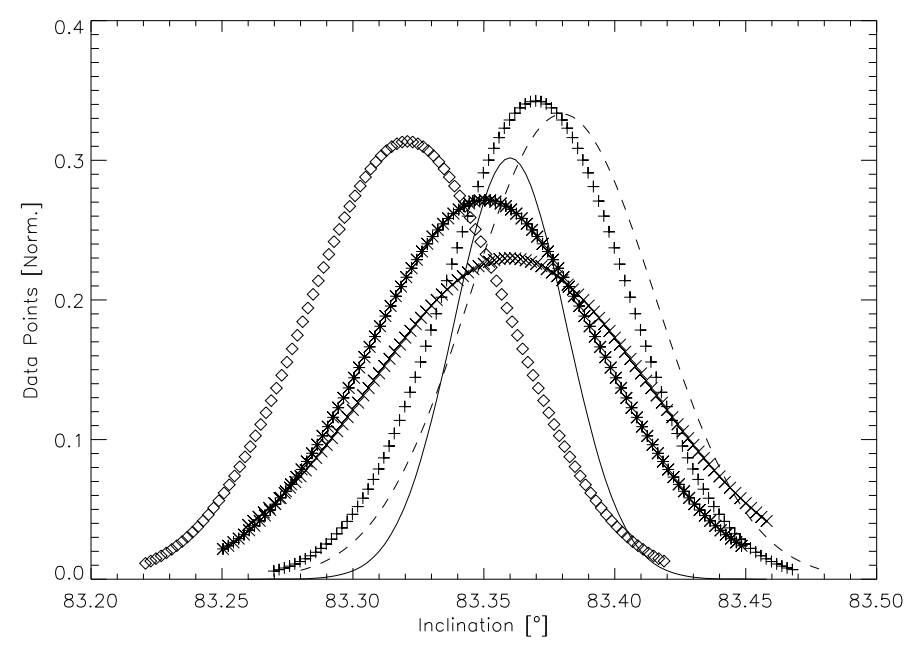

Fig. 4. Inclination distribution derived 1000 Monte Carlo simulations for the multi-band BUSCA observations data in the $I$ (crosses), $y$ (stars), $b$ (diamonds) and $v$ ( $\times$-symbol) filter bands; the mean inclination distribution from the four BUSCA lightcurves is shown as solid line, the inclination distribution derived from the OLT data taken in April 2009 is also shown for comparison (dashed line).

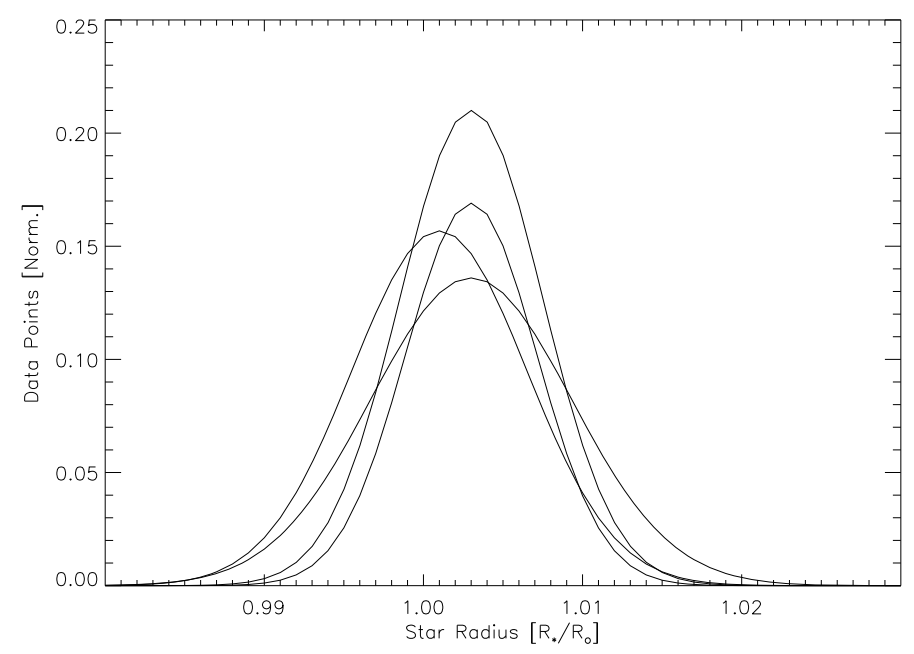

Fig. 5. Stellar radius distribution $\left(R_{*}\right)$, derived from 1000 Monte Carlo simulations in four different filters (from higher to lower peak, $I, y, b$ and $v$ filter respectively). The overlap of the curves shows that all colors can be explained with the same stellar radius as suggested by theory (see Neckel 1995).

for the inclination we assumed for, first, the value $i=83.57^{\circ}$ (as derived by Holman et al. for their 2006 data) and the value $i=83.36^{\circ}$ (this paper for the 2009 data). The fit results (in terms of obtained $\chi^{2}$ values) are summarised in Table 4, which shows that for all (independent) data sets the lower inclination values yield smaller $\chi^{2}$-values; for some filter pairs the thus obtained improvement is extremely significant. 


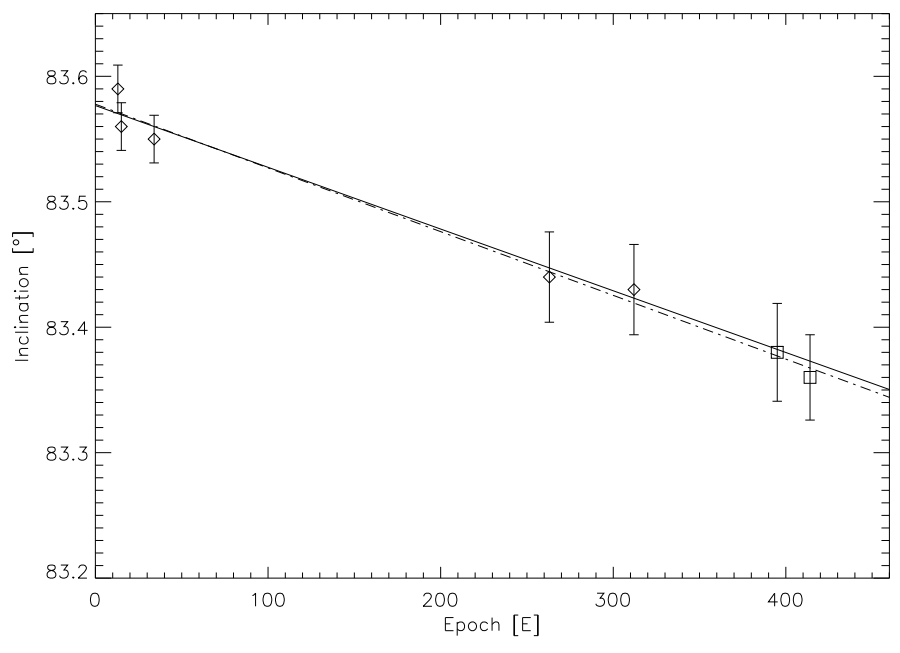

Fig. 6. Epoch versus inclination together with a linear fit to the currently available data; the diamond points are those taken in 2006 by Holman et al. (2007), and those taken in 2008 and reported in Paper I. The square points are derived from our new observations taken in April and May 2009. The solid lines showing two linear fits, from the first paper (dashed line) and the fit from the present paper (solid line).

\subsection{Inclination changes}

In Fig. 6 we plot all our current inclination vs. epoch data together with a linear fit to all data; a formal regression analysis yields for the time evolution of the inclination $\left(i=i_{\mathrm{o}}+a\right.$. (Epoch), $\left.i_{\mathrm{O}}=83.5778, a=0.00051\right)$. In Paper I we noted the inclination decrease and predicted inclination values below the first transit threshold $\left(i_{\min , 1}<83.417^{\circ}\right)$ after October 2008 . Both the new OLT data set and all BUSCA channel observations yield inclinations below the first transit threshold. While the error in a given transit light curve is typically on the order of $0.04^{\circ}$ for $i$, we consider it quite unlikely that 5 independent measurements all yield only downward excursions. We therefore consider the decrease in inclination between fall 2008 and spring 2009 as significant, conclude that the inclination in the TrES-2 system is very likely below the first transit threshold, and predict the inclinations to decrease further; also, the transit depths should become more and more shallow since the exoplanet eclipses less and less stellar surface.

\subsection{Period changes}

The observed change in orbit inclination is in marked contrast to the period of TrES-2b. While possible TTVs in TrES-2b have been studied by Rabus et al. (2009) we investigate the longterm stability of the period of TrES-2b. From our seven transit measurements (plus five more data points of Rabus et al. 2009) spanning about $\sim 400$ eclipses we created a new O-C diagram (cf., Fig. 7); note that we refrained from using the transit times dicussed by Raetz et al. (2009), since these transits were taken with rather inhomogeneous instruments and sometimes only partial transit coverage. For our fit we used a modified epoch equation $\mathrm{HJD}_{\mathrm{c}}=\mathrm{HJD}_{\mathrm{o}}+E \cdot P$, where we set $P=P_{\mathrm{o}}+\dot{P}\left(t-\mathrm{HJD}_{\mathrm{o}}\right)$ and explicitly allow a non-constant period $P$. We apply a $\chi^{2}$ fit to find the best fit values for $\dot{P}, P_{\mathrm{o}}$, and $\mathrm{HJD}_{\mathrm{o}}$. With this approach we find a best fit period change of $\dot{P}=5 \times 10^{-9}$, however, carrying out the same analysis keeping a fixed period shows that the fit improvement due to the introduction of a non-zero $\dot{P}$ is insignificant. We then find as best fit values $P_{\mathrm{o}}=2.47061$ and $\mathrm{HJD}_{\mathrm{o}}=2453957.6350$ conforming to the values derived by

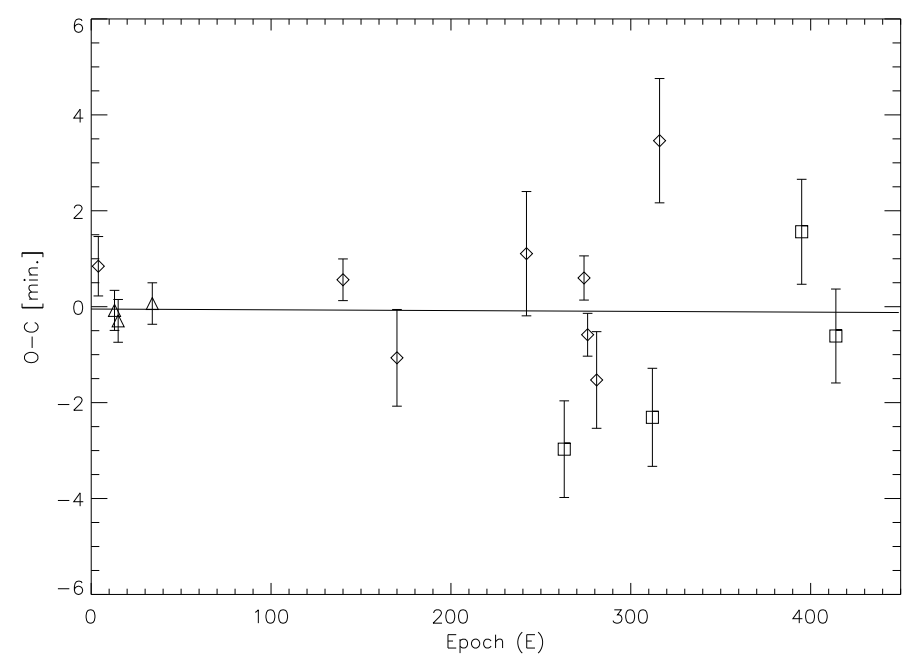

Fig. 7. O-C values versus epoch including the transits observed by Holman et al., Rabus et al. and our data denoted by triangles, squares and diamonds respectively.

Rabus et al. (2009). Thus, the period of TrES-2b is constant, and any possible period change over the last three years must be less than about $1 \mathrm{~s}$.

\section{Theoretical implications of observed inclination change}

The results of our data presented in the preceding sections strenghten our confidence that the observed inclination changes do in fact correspond to a real physical phenomenon. Assuming now the reality of the observed inclination change of $\Delta i \sim$ $0.075^{\circ} / \mathrm{yr}$, given the constancy of the period and the absence of TTVs at a level of $\approx 100 \mathrm{~s}$ we discuss in the following a physical scenario consistent with these observational findings. We specifically argue that the apparent inclination change should be interpreted as a nodal regression and then proceed to examine an oblate host star and the existence of an additional perturbing object in the system as possible causes for the change of the orbital parameters of Tres- $2 b$.

\subsection{Inclination change or nodal regression?}

It is important to realize that the reported apparent change of inclination refers to the orientation of the TrES-2 orbit plane with respect to the observer's tangential plane. It is well known that the $z$-component of angular momentum for orbits in an azimuthally symmetric potential is constant, resulting in a constant value of inclination. An oblate star (cf., Sect. 4.2) or the averaged potential of a third body (cf., Sect. 4.3) naturally lead to such potentials, thus yielding orbits precessing at (more or less) constant inclination as also realized by Miralda-Escudé (2002). Such a precession would cause an apparent inclination change, however, physically this would have to be interpreted as nodal regression at fixed orbit inclination. To interpret the observations, one has to relate the rate of nodal regression to the rate of apparent inclination change.

Consider a (massless) planet of radius $R_{\mathrm{p}}$ orbiting a star of radius $R_{*}$ at some distance $d$. Let the planet's orbit lie in a plane with a fixed inclination $i$ relative to the $x-y$ plane, which we take as invariant plane. Let an observer be located in the $x-z$ plane with some elevation $\gamma$, reckoned from the positive $x$-axis. The 


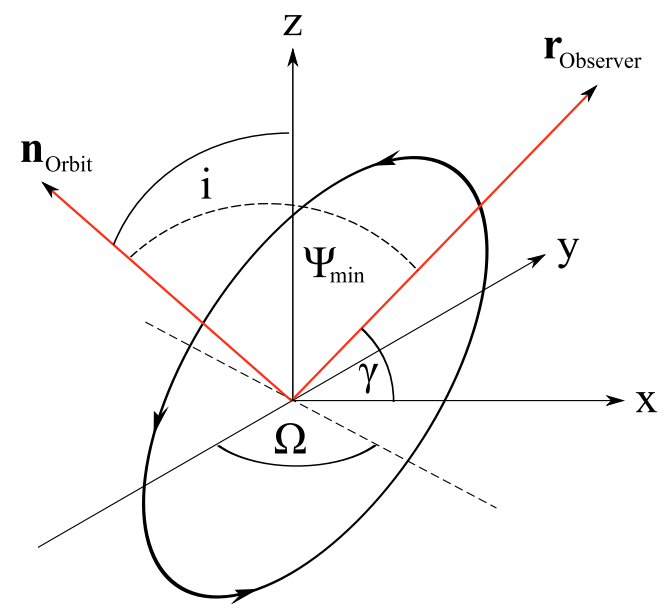

Fig. 8. System geometry (see text for details): the orbit plane with normal vector $\boldsymbol{n}_{\text {Orbit }}$ is inclined relative to the $x-y$ plane by an inclination $i$, the observer (towards $\boldsymbol{r}_{\text {Observer }}$ ) is within the $x-z$ plane.

line of the ascending node in the $x-y$ plane is denoted by the angle $\Omega$, with $\Omega=0$ implying the ascending node pointed along the negative $y$-axis (see Fig. 8). Let in the thus defined geometry the angle $\Psi$ denote the angle between planet and observer as seen from the central star. For each system configuration defined by the angles $(\gamma, i, \Omega)$ there is a minimal angle $\Psi_{\text {min }}$ between orbit normal and observer obtained in each planetary orbit, which can be computed from

$$
\cos \Psi_{\min }=\boldsymbol{n}_{\text {Orbit }} \cdot \boldsymbol{r}_{\text {Observer }}=-\cos \gamma \sin i \cos \Omega+\sin \gamma \cos i \text {. }
$$

A transit takes place when

$\left|\cos \Psi_{\min }\right| \leq\left(R_{p}+R_{*}\right) / d$,

and from the geometry it is clear that the observed inclination $i_{\text {obs }}$, i.e., the parameter that can be derived from a transit light curve is identical to $\Psi_{\min }$. Setting then $\Psi_{\text {min }}=i_{\text {obs }}$ and differentiating Eq. (1) with respect to time we obtain

$\frac{\mathrm{d} i_{\mathrm{obs}}}{\mathrm{d} t}=-\frac{\sin \Omega \cos \gamma \sin i}{\sin i_{\mathrm{obs}}} \frac{\mathrm{d} \Omega}{\mathrm{d} t}$.

Equation (3) relates the nodal regression of the orbit to its corresponding observed apparent rate of inclination change $\mathrm{d} i_{\mathrm{obs}} / \mathrm{d} t$, given the fixed inclination $i$ relative to the $x-y$ plane. Since transit observations yield very precise values of $i_{\mathrm{obs}}$, the required ascending node $\Omega$ and its rate of change can be computed, once the orbit geometry through the angles $\gamma$ and $i_{\text {obs }}$ is specified. In Fig. 9 we show a contour plot of the linear coefficient of Eq. (3) between nodal regression and observed inclination change as a function of orbit geometry. Note that the apparent change of inclination due to the nodal regression does vanish for $i=0$. Physically it is clear that a perturbing planet in a coplanar orbit cannot exercise a torque and therefore cannot cause the observed inclination variation. We will therefore always assume $i \neq 0$ in the following.

\subsection{Oblate host star}

We first consider the possibility that the TrES-2 host star is oblate. The motion of a planet around an oblate host star is equivalent to that of an artifical satellite orbiting the Earth, a problem intensely studied over the last decades and well understood Connon Smith (2005). The potential $U(r, \phi)$ of an axisymmetric

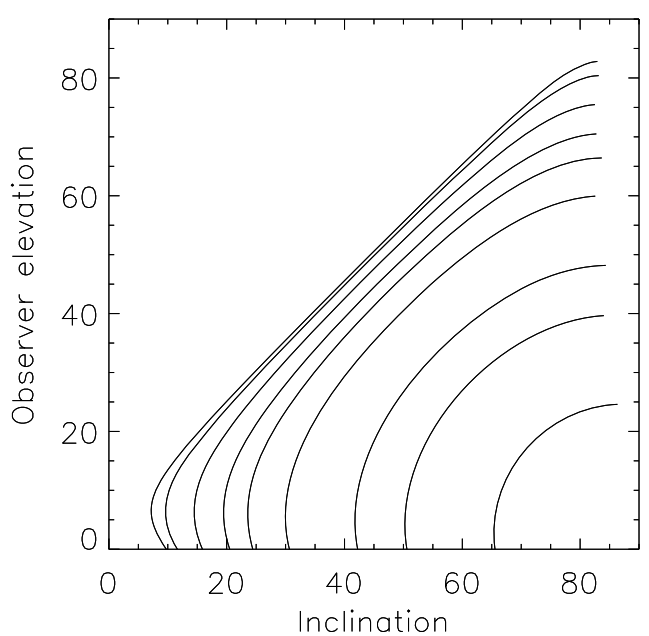

Fig. 9. Linear coefficient of Eq. (3) between nodal regression and observed inclination change as a function of view geometry (cf., Eq. (3)), computed for $i_{\mathrm{obs}}=83.38^{\circ}$ as applicable for TrES-2; the plotted contour levels denote values of 1.1, 1.3, 1.5, 2., 2.5, 5., 4., 6., 8. from right bottom up.

body of mass $M$ and radius $R$ can be expressed as a power series involving the so-called harmonic coefficients. In second order one approximates the potential $U(r, \phi)$ as

$U(r, \phi)=\frac{G M}{r}\left[1-J_{2}\left(\frac{R}{r}\right)^{2} \frac{1}{2}\left(3 \sin ^{2} \phi-1\right)\right]$,

where $r$ is the radial distance from the body's center, $\phi$ is latitude above the equator and $G$ denotes the gravitational constant. Clearly, the perturbing term in the potential is proportional to $J_{2}$ and a perturbation calculation yields as first order secular perturbation the angular velocity of the ascending node as

$\frac{\mathrm{d} \Omega}{\mathrm{d} t}=-\frac{3}{2} \frac{J_{2} R^{2}}{\left(1-e^{2}\right)^{2}} \cos i\left(\frac{2 \pi}{P}\right)^{7 / 3} \frac{1}{\left(G M_{\mathrm{total}}\right)^{2 / 3}}$,

where $e$ and $i$ denote the eccentricity and inclination of the orbiting body, $P$ its period, $M_{\text {total }}$ the sum of the masses of planet and host star and the validity of Kepler's third law has been assumed.

Interpreting the observed inclination change as nodal regression due to an oblate host star, we can compute a lower bound on the required harmonic coefficient $J_{2}$ assuming $e=0$. Therefore, we combine Eqs. (3) and (5) to obtain an expression for $J_{2}$. Excluding pathological cases like $i, \Omega=0$ and $\gamma=\pi / 2$ and neglecting the planetary mass in Eq. (5) we find for any given set of parameters $(\Omega, \gamma, i)$

$J_{2} \geq J_{2}^{\min }=\frac{2}{3} \frac{\mathrm{d} i_{\mathrm{obs}}}{\mathrm{d} t}\left(\frac{P}{2 \pi}\right)^{7 / 3} \frac{\left(G M_{\mathrm{host}}\right)^{2 / 3}}{R^{2}} \sin i_{\mathrm{obs}}$,

where $M_{\text {host }}$ denotes the mass of the host star. Mass, radius, inclination and period of the TrES-2 system are well known, and using the measured nodal regression we find $J_{2, \mathrm{TrES}-2} \approx 1.4 \times 10^{-4}$, i.e., a value smaller than that of the Earth $\left(J_{2, \oplus}=0.00108\right)$ by an order of magnitude, but considerably larger than that of the Sun, which is usually taken as $J_{2, \odot} \approx 3-6 \times 10^{-7}$ Rozelot et al. (2001). Since the host star of TrES-2 is a slow rotator very similar to the Sun Sozzetti et al. (2007), we expect similarly small $J_{2}$ values in contrast to our requirements. We therefore conclude that oblateness of the host star cannot be the cause for the observed orbit variations. 


\subsection{Perturbation by a third body}

An alternative possibility to explain the observed orbit variations of the TrES-2 exoplanet would be the interaction with other planets in the system. Let us therefore assume the existence of such an additional perturbing planet of mass $m_{\mathrm{p}}$, circling its host star of mass $m_{0}$ with period $P_{\mathrm{p}}$ at distance $r_{\mathrm{p}}$ located further out compared to the known transiting TrES-2 exoplanet. This three-body problem has been considered in past in the context of triple systems (Khaliullin et al. 1991; Li 2006) and the problem of artificial Earth satellites, whose orbits are perturbed by the Moon. In lowest order, the perturbing gravitational potential $R_{2}$ onto the inner planet with mass $m$ and distance $r$ is given by the expression

$R_{2}=\frac{m_{\mathrm{p}}}{m_{\mathrm{p}}+m_{o}}\left(\frac{2 \pi a}{P_{\mathrm{p}}}\right)^{2}\left(\frac{a_{\mathrm{p}}}{r_{\mathrm{p}}}\right)^{3}\left(\frac{r}{a}\right)^{2} \frac{3 \cos ^{2} S-1}{2}$,

where the angle $S$ denotes the elongation between the perturbed and perturbing planet as seen from the host star, $a$ and $a_{\mathrm{p}}$ denote their respective semi-major axes and the validity of Kepler's third law has been assumed. Note that in this approach the perturbed body is assumed to be massless, implying that its perturbations onto the perturbing body are ignored. Next one needs to insert the orbital elements of the two bodies and, since we are interested only in secular variations, average over both the periods of the perturbed and perturbing planet. This is the so-called double-averaging method (Broucke 2003), which, however, in more or less the same form has also been applied by Li (2006) and Kovalevsky (1967). Denoting by $e$ the eccentricity of the perturbed planet, by $\omega$ the longitude of the periastron and by $i$ the angle between the two orbital planes, one obtains after some lengthy computation (see Kovalevsky 1967)

$R_{2}=\frac{m_{\mathrm{p}}}{m_{\mathrm{p}}+m_{\mathrm{o}}}\left(\frac{\pi a}{2 P_{\mathrm{p}}}\right)^{2} \times K_{0}(i, e, \omega)$,

with the auxiliary function $K_{0}(i, e, \omega)$ given by

$K_{0}(e, i, \omega)=\left(6 \cos ^{2} i-2\right)+e^{2}\left(9 \cos ^{2} i-3\right)+15 e^{2} \sin ^{2} i \cos 2 \omega$.

The partial derivatives of $R_{2}$ with respect to the orbital elements are needed in the so-called Lagrangian planetary equations to derive the variations of the orbital elements of the perturbed body. One specifically finds for the motion of the ascending node

$\frac{\mathrm{d} \Omega}{\mathrm{d} t}=\frac{m_{\mathrm{p}}}{m_{\mathrm{p}}+m_{\mathrm{o}}} \frac{3 \pi}{4} \frac{P}{P_{\mathrm{p}}^{2}} \frac{\cos i}{\sqrt{1-e^{2}}} \times K_{1}(e, \omega)$,

where the auxiliary function $K_{1}(e, \omega)$ is defined through

$K_{1}(e, \omega)=5 e^{2} \cos 2 \omega-3 e^{2}-2$

and for the rate of change of inclination

$\frac{\mathrm{d} i}{\mathrm{~d} t}=-e^{2} \frac{m_{\mathrm{p}}}{m_{\mathrm{p}}+m_{\mathrm{o}}} \frac{15 \pi}{8} \frac{P}{P_{\mathrm{p}}^{2}} \frac{1}{\sqrt{1-e^{2}}} \sin (2 i) \sin (2 \omega)$.

As is obvious from Eq. (11), the rate of change of inclination in low eccentricity systems is very small and we therefore set $e=0$. Assuming next a near coplanar geometry, i.e., setting $\cos i \approx 1$, we can simplify Eq. (9) as

$\frac{\mathrm{d} \Omega}{\mathrm{d} t}=-\frac{m_{\mathrm{p}}}{m_{\mathrm{p}}+m_{\mathrm{o}}} \frac{3 \pi}{2} \frac{P}{P_{\mathrm{p}}^{2}}$.

If we assume a host star mass and interpret the observed inclination change as the rate of nodal regression via Eq. (3), Eq. (12) relates the unknown planet mass $m_{\mathrm{p}}$ to its orbital period $P_{\mathrm{p}}$.

\subsubsection{Sanity check: application to the solar system}

The use of Eq. (12) involves several simplifications. Thus, it is legitimate to ask, if we are justified in expecting Eq. (12) to describe reality. As a sanity check we apply Eq. (12) to our solar system. Consider first the motion of the Moon around the Earth, i.e., $P=27.3 \mathrm{~d}$, which is perturbed by the Sun, i.e., $P_{\mathrm{p}}=365.25 \mathrm{~d}$. Since for that case our nomenclature requires $m_{\mathrm{p}} \gg m_{\mathrm{o}}$ and $i \sim 5.1^{\circ}$, we find from Eq. (12) a time of 17.83 years for the nodes to complete a full circle, which agrees well with the canonical value of 18.6 years for the lunar orbit. In the lunar case it is clear that the Sun with its large mass and close proximity (compared to Jupiter) is by far the largest perturber of the Earth-Moon two-body system and this situation is exactly the situation described by theory.

Consider next the the perturbations caused by the outer planets of our solar system. Considering, for example, Venus, we can compute the perturbations caused by the planets Earth, Mars, Jupiter, Saturn and Uranus. Since the perturbation strength scales by the ratio $m_{\mathrm{p}} P_{\mathrm{p}}^{-2}$, we can set this value to unity for the Earth and compute values of 0.03, 2.26, 0.11 and 0.002 for Mars through Uranus respectively. So clearly, Venus is perturbed by several planets, but the perturbations by Jupiter are strongest. We therefore expect that our simple approach is not appropriate. We further note that among the outer solar system planets long period perturbations and resonances occur, which are not described by Eq. (12). If we nevertheless compute the nodal regression for Venus caused by Jupiter using Eq. (12), we find a nodal regression of $0.1 \%$ cty for Venus, and $0.3 \%$ cty for Mars. Using the orbital elements computed by Simon et al. (1994) and calculating the nodal regressions of Venus and Mars in the orbit plane of Jupiter we find values smaller than the true values, but at least, they computed values in the right order of magnitude and do not lead to an overprediction of the expected effects.

\subsubsection{Sanity check: application to V 907 Sco}

From archival studies Lacy et al. (1999) report the existence of transient eclipses in the triple star V 907 Sco. According to Lacy et al. (1999) this system is composed out of a short-period $\left(P_{\text {short }}=3.78\right.$ days $)$ binary containing two main sequence stars of spectral type $\sim \mathrm{A} 0$ and mass ratio 0.9 , orbited by a lower mass third companion $\left(P_{\text {long }}=99.3\right.$ days $)$, of spectral type mid-K or possibly even a white dwarf. The close binary system showed eclipses from the earliest reported observations in 1899 until about 1918, when the eclipses stopped; eclipses reappeared in 1963 and were observed until about 1986. Interpreting the appearance of eclipses due to nodal regression, Lacy et al. (1999) derive a nodal period of 68 years for V 907 Sco. Using Eq. (12) and assuming a mass of $2 M_{\odot}$ for the host $\left(m_{\mathrm{o}}\right)$ and a mass of $0.5 M_{\odot}$ for the companion $\left(m_{\mathrm{p}}\right)$, we compute a nodal regression period of 47.6 years, which agrees well with the nodal period estimated by Lacy et al. (1999). We therefore conclude that Eq. (12) also provides a reasonable description of the transient eclipse observations of V 907 Sco.

\subsubsection{Application to TrES 2}

Applying now Eq. (12) to the TrES-2 exoplanet we express the period of the (unknown) perturbing planet as a function of its also unknown mass through

$P_{\mathrm{p}}=\sqrt{\frac{m_{\mathrm{p}}}{m_{\mathrm{p}}+m_{\mathrm{o}}} \frac{3 \pi P}{2} \frac{1}{\mathrm{~d} \Omega / \mathrm{d} t}}$. 


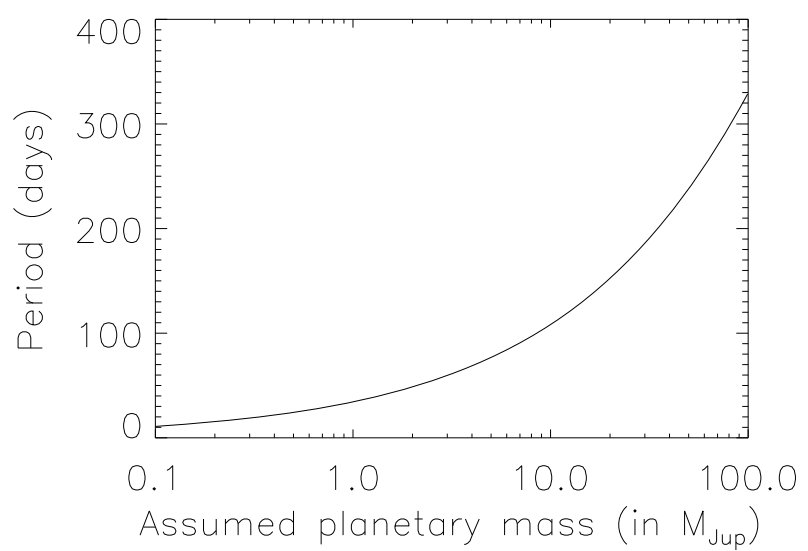

Fig. 10. Period of hypothesized second planet vs. mass assuming a linear coefficient of unity in Eq. (3) and near coplanarity.

Since the host star mass and the nodal regression are known, the perturbing mass is the only remaining unknown; we note that Eq. (13) should be correct, as long as there is only one dominant perturber in the system with a low-eccentricity orbit sufficiently far away from the known close-in transiting planet. In Fig. 10 we plot the expected perturber period $P_{\mathrm{p}}$ as a function of $m_{\mathrm{p}}$ (in Jupiter masses) for $m_{\mathrm{o}}=1 M_{\odot}$ and the measured rate of nodal regression assuming a linear coefficient of unity in Eq. (3). Assuming ad hoc a mass of about one Jupiter mass for this perturber and taking into account that a factor of a few is likely (cf., Fig. 9), we find that periods of 50 to 100 days are required to explain the observed nodal regression in TrES-2. Such an additional planet should be relatively easily detectable with RV studies. Daemgen et al. (2009) report the presence of a faint companion about one arcsec away from TrES-2. Assuming this companion to be physical, a spectral type between K4.5 and K6, a mass of about $0.67 M_{\odot}$ at a distance of $230 \mathrm{AU}$ with a period of 3900 years follow. Computing with these numbers the maximally expected nodal regression from Eq. (12), one finds values a couple of orders of magnitude below the observed values. We thus conclude that this object cannot be the cause of the observed orbit variations. On the other hand, this companion, again if physical, makes TrES-2 particularly interesting because it provides another cases of a planet/planetary system in a binary system, and eventually the orbit planes of binary system and the planet(s) can be derived.

\subsection{Transit timing variations by a putative perturber}

A perturbing second planet capable of causing fast nodal precession on the transiting planet is also expected to induce shortterm periodic variations of its orbital elements. In addition to the secular precession of the node of the orbit we would thus expect to see short-term transit timing variations (TTVs), periodic variations of the mid-transit times (Holman \& Murray 2005; Agol et al. 2005). Just as nodal regression, the TTV signal can be used to find and characterize planetary companions of transiting exoplanets. Rabus et al. (2009) carefully analyzed eight transit light curves of TrES-2 over several years. However, they were unable to detect any statistically significant TTV amplitudes in the TrES-2b light curves above about $50 \mathrm{~s}$; cf. their Fig. 10. Therefore, the existence of perturbing objects leading to TTVs on the scale of up to about $50 \mathrm{~s}$ are consistent with actual observations. Putting it differently, the orbital parameters of any perturbing object causing the nodal precession of the orbit

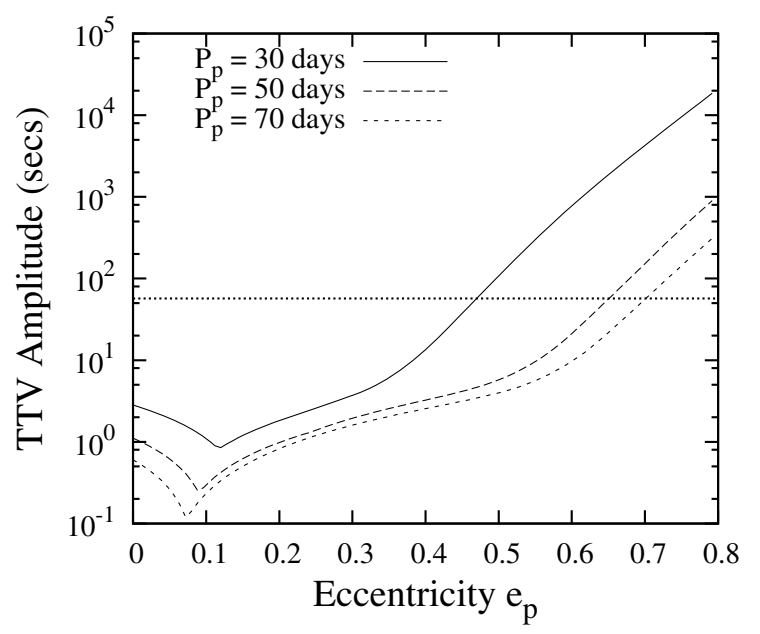

Fig. 11. Amplitude of expected Transit Timing Variations (TTVs) in the TrES-2 system. The perturber is assumed to have $m_{\mathrm{p}}=1 M_{\text {Jup }}$. Its eccentricity $e_{\mathrm{p}}$ and period $P_{\mathrm{p}}$ are varied within plausible ranges. The orbit of TrES- 2 is assumed to be circular. The vertical dotted line marks the best fitting TTV signal found by Rabus et al. (2009) of $57 \mathrm{~s}$.

should yield a TTV amplitude below that and hence remain undetactable in the presently existing data.

To analyze the mutual gravitational influence of a perturbing second planet in the system on TrES-2, we have to treat the classical three-body problem of celestial mechanics. Instead of direct $N$-body integrations of the equations of motion we use an alternative method based on analytic perturbation theory developed and extensively tested by Nesvorný \& Morbidelli (2008) and Nesvorný (2009). Outside possible mean-motion resonances their approach allows for a fast computation of the expected TTV amplitude given a combination of system parameters. As input we have to specify the orbital elements and masses of both planets. Consistent with the observations we assume TrES-2 to be in a circular orbit around its host star, while we allow for different eccentricities $e_{\mathrm{p}}$ and periods $P_{\mathrm{p}}$ of the perturber, which we assume to be of Jovian mass; since the TTV amplitudes scale nearly linearly with the perturber mass, we confine our treatment to $m_{p}=1 M_{\text {Jup }}$; all other orbital elements are set to zero. This is justified as these parameters in most cases do not lead to a significant amplification of the TTV signal (see Nesvorný 2009, for a detailed discussion of the impact of these orbital elements). The resulting TTV amplitude for different reasonable orbit configurations (given the observed secular node regression as discussed above) of the system of $P_{\text {pert }}=30,50$ and 70 days is plotted vs. the assumed eccentricity in Fig. 11; the currently available upper limit to any TTV signal derived by Rabus et al. (2009) is also shown. As is obvious from Fig. 11, a Jovian-mass perturber at a distance required to impose the observed secular changes (period of 50-100 days) leads to a TTV signal well below the current detection limit for all eccentricities $e_{\mathrm{p}}$ as long as $e_{\mathrm{p}} \lesssim 0.4$. We therefore conclude that a putative perturbing Jovian-mass planet with a moderate eccentricity and with a period between 30-70 days would not yield any currently detectable TTV signal and would therefore be a valid explanation for the observed inclination change in the TrES-2 system.

\section{Conclusions}

In summary, our new observations taken in the spring of 2009 confirm the smaller transit durations reported in Paper I and suggest an even further decrease. With our simultaneously taken 
multicolor BUSCA data we demonstrate that the recorded multicolor lightcurves can be consistently modelled with a reasonable set of limb darkening coefficients, and that there is no need to fit the limb darkening coefficients to any particular light curve. An error in the description of the limb darkening therefore appears thus as an unlikely cause of the observed inclination changes. Also as expected, the obtained stellar radius is independent of the wavelength band used, demonstrating the internal self-consistency of our modelling.

As to the possible causes for the observed apparent orbit inclination change in TrES-2 we argue that the apparent observed inclination change is very likely caused by nodal regression. The assumption of an oblate host star leads to implausibly large $J_{2}$ coefficients, we therefore favor an explanation with a third body. We argue that Eq. (12) is a reasonable approximation for the interpration of the observed inclination changes; applying it to the TrES-2 system, we find that a planet of one Jovian mass with periods between 50-100 days would suffice to cause the observed inclination changes, while at the same time yield TTVs with amplitudes well below the currently available upper limits.

The assumption of such an additional planet in the TrES-2 system is entirely plausible. First of all, if it is near coplanar with TrES-2b, it would not cause any eclipses and therefore remain undetected in transit searches. Next, an inspection of the exosolar planet data base maintained at www.exoplanet.eu reveals a number of exoplanet systems with properties similar to those postulated for TrES-2, i.e., a close-in planet together with a massive planet further out: In the Gl 581 system there is a 0.02 Jupiter-mass planet with a period of 66 days, and in fact a couple of similarly massive planets further in with periods of 3.1, 5.4 and 12.9 days respectively; in the system HIP 14810 there is a close-in planet with a 6.6 day period and a somewhat lighter planet with a period of 147 days, in the HD 160691 system the close-in planet has a period of 9.6 days and two outer planets with Jupiter masses are known with periods of 310 and 643 days. It is also clear that in these systems nodal regression changes must occur, unless these systems are exactly coplanar, which appears unlikely. Therefore on longer time scales the observed orbit inclination in these systems must change, but only in transiting systems the orbit inclination can be measured with sufficient accuracy. Because of its apparent inclination change TrES-2b is clearly among the more interesting extrasolar planets. If the system continues its behavior in the future the transits of TrES-2b will disappear. Fortunately, within the first data set of the Kepler mission $\sim 30$ transits should be covered. From our derived inclination change rate of $\Delta i \sim 0.075^{\circ} / \mathrm{yr}$ this corresponds to an overall change of $\Delta i \sim 0.015^{\circ}$ in this first data set, which ought to be detectable given the superior accuracy of the space-based Kepler photometry. As far as the detection of our putative second planet is concerned, RV methods appear to be more promising than a search for TTVs, unless the orbital eccentricities are very large.

Acknowledgements. The paper is based on observations collected with the $2.2 \mathrm{~m}$ telescope at the Centro Astronómico Hispano Alemán (CAHA) at Calar Alto (Almería, Spain), operated jointly by the Max-Planck Institut für Astronomie and the Instituto de Astrofísica de Andalucía (CSIC). The authors thank H.Poschmann and the BUSCA team for their tremendous work on the new controller and CCD system. D.M. was supported in the framework of the DFGfunded Research Training Group "Extrasolar Planets and their Host Stars" (DFG 1351/1). S.S. acknowledges DLR support through the grant 50OR0703.

\section{References}

Agol, E., Steffen, J., Sari, R., \& Clarkson, W. 2005, MNRAS, 359, 567 Alonso, R., Barbieri, M., Rabus, M., et al. 2008, A\&A, 487, L5 Broucke, R. A. 2003, J. Guidance Control Dynamics, 26, 27

Claret, A. 2004, A\&A, 428, 1001

Connon Smith, R. 2005, The Observatory, 125, 341

Daemgen, S., Hormuth, F., Brandner, W., et al. 2009, A\&A, 498, 567

Holman, M. J., \& Murray, N. W. 2005, Science, 307, 1288

Holman, M. J., Winn, J. N., Latham, D. W., et al. 2007, ApJ, 664, 1185

Howell, S. B., \& Everett, M. E. 2001, in Third Workshop on Photometry, ed. W. J. Borucki, \& L. E. Lasher, 1

Khaliullin, K. F., Khodykin, S. A., \& Zakharov, A. I. 1991, ApJ, 375, 314

Kovalevsky, J. 1967, in Les Nouvelles Méthodes de la Dynamique Stellaire, 221 Lacy, C. H. S., Helt, B. E., \& Vaz, L. P. R. 1999, AJ, 117, 541

Li, L. 2006, AJ, 131, 994

Miralda-Escudé, J. 2002, ApJ, 564, 60

Mislis, D., \& Schmitt, J. H. M. M. 2009, A\&A, 500, L45

Neckel, H. 1995, Sol. Phys., 156, 7

Nesvorný, D. 2009, ApJ, 701, 1116

Nesvorný, D., \& Morbidelli, A. 2008, ApJ, 688, 636

O’Donovan, F. T., Charbonneau, D., Mandushev, G., et al. 2006, ApJ, 651, L61

O'Donovan, F. T., Charbonneau, D., Harrington, J., et al. 2009, in IAU Symp., 253,536

Rabus, M., Deeg, H. J., Alonso, R., Belmonte, J. A., \& Almenara, J. M. 2009, A\&A, 508, 1011

Raetz, S., Mugrauer, M., Schmidt, T. O. B., et al. 2009, Astron. Nachr., 330, 459

Rozelot, J. P., Godier, S., \& Lefebvre, S. 2001, Sol. Phys., 198, 223

Simon, J. L., Bretagnon, P., Chapront, J., et al. 1994, A\&A, 282, 663

Sozzetti, A., Torres, G., Charbonneau, D., et al. 2007, ApJ, 664, 1190 